\title{
Frequency Diverse Array Target Localization Based on IPSO-BP
}

\author{
Qinghua Liu $\mathbb{D},{ }^{1}$ Kai Ding $\mathbb{D}{ }^{2}{ }^{2}$ Bingsen $W u,{ }^{1}$ and Quanmin $X i e^{3,4}$ \\ ${ }^{1}$ State and Local Joint Engineering Research Center for Satellite Navigation and Location Service, \\ Guilin University of Electronic Technology, Guilin 541004, China \\ ${ }^{2}$ Science and Technology on Near-Surface Detection Laboratory, Wuxi 214035, China \\ ${ }^{3}$ Ordnance NCO Academy, Army Engineering University, Wuhan 430075, China \\ ${ }^{4} \mathrm{Hubei}$ (Wuhan) Explosions and Blasting Technology Institute of Jianghan University, Wuhan 430056, China \\ Correspondence should be addressed to Kai Ding; winfast113@sina.com
}

Received 29 May 2020; Revised 21 July 2020; Accepted 13 August 2020; Published 27 August 2020

Academic Editor: Mauro Parise

Copyright (C) 2020 Qinghua Liu et al. This is an open access article distributed under the Creative Commons Attribution License, which permits unrestricted use, distribution, and reproduction in any medium, provided the original work is properly cited.

For the traditional target localization algorithms of frequency diverse array (FDA), there are some problems such as angle and distance coupling in single-frequency receiving FDA mode, large amount of calculation, and weak adaptability. This paper introduces a good learning and predictive method of target localization by using BP neural network on FDA, and FDA-IPSO-BP neural network algorithm is formed. The improved particle swarm optimization (IPSO) algorithm with nonlinear weights is developed to optimize the neural network weights and biases to prevent BP neural network from easily falling into local minimum points. In addition, the decoupling of angle and distance with single frequency increment is well solved. The simulation experiments show that the proposed algorithm has better target localization effect and convergence speed, compared with FDA-BP and FDA-MUSIC algorithms.

\section{Introduction}

Since the frequency diverse array (FDA) was first proposed in 2006 [1], many scholars from all over the world have conducted research on it. Different from the traditional phased array, FDA introduces a small frequency increment on the array elements $[2,3]$, which induces the beam pattern curved in space and extends the spatial degrees of freedom. In [4], FDA was applied to the detection of ground moving targets by forward-looking radars. Compared with the traditional phased array radar, it can suppress the distance fuzzy clutter to some extent. In [5], FDA was first applied to synthetic aperture radar for high resolution imaging, and it was verified by simulation results that FDA can improve the imaging resolution of azimuth direction and range direction of synthetic aperture radar. In [6], a target localization method was proposed to suppress the interference information in the echo with the nonuniform frequency increment determined by the genetic optimization algorithm, and the position of the targets was estimated by MUSIC algorithm. FDA-MIMO radar technology was studied in [7-9], and it was concluded that the FDA-MIMO radar has a good improvement on side lobe suppression and target detection performance. In [10], the multidimensional information (distance, angle, and amplitude) estimation method of FDAMIMO radar was proposed based on sparse iteration. By optimizing the objective function of the weighted norm, the distance, angle, and amplitude information of the target were obtained by sparse iterative optimization. In [11], a target localization algorithm with the compressed sensing sparse representation was proposed. The target localization problem was described as a cost function under the sparse representation framework, which was optimized by the convex optimization tool. The non-zero element index in the obtained sparse weight vector mapped the orientation and distance information of the target. In [12], FDA and waveform multiplexing were applied to bistatic radar systems. In [13], a bistatic FDA-MIMO radar was introduced, and nonlinear frequency increment was used to overcome the coupling problem of DOD and distance parameters. The rotation invariance technique and parallel factor algorithm were proposed correspondingly, which can reduce the 
complexity of computational and three-dimensional spectral peak searching.

All the above methods are based on the pure mathematical models to perform a large number of operations to obtain the final result, which causes the adaptability to the environment to be poor; meanwhile, the real-time application is unachievable. In this paper, by introducing the neural network method, the problem of the angle-distance coupling is solved well in the single-frequency receiving FDA mode with single frequency increment, and the trained model has certain environmental adaptability with lower calculation amount $[14,15]$.

The neural network algorithm has good learning and predictability through the training model, which was applied to target location. In [16], a single linear array combined with appropriately trained linear vector quantization (LVQ) artificial neural networks is used to achieve two-dimensional direction of arrival (2D-DOA) estimation with elevation and azimuth angle estimates. In [17], a fast estimation method based on LVQ neural network for two-dimensional DOA is proposed. By using the symmetry of the UCA array, two different elevation and azimuth data sets were provided, and a LVQ neural network was sequentially trained on each data set to estimate the elevation and the azimuth angles, respectively.

The above documents $[16,17]$ derived the azimuth and elevation angles of the target from the characteristics of the traditional phased array on the angle information. The distance of the target was obtained by introducing the orientation values into the signal model, which leads to a further increase of computation.

This paper develops a target localization algorithm by combining IPSO (improved particle swarm optimization), BP (backpropagation) neural network, and FDA, named as FDA-IPSO-BP, by which the coupling problem of angle and distance in the FDA can be avoided; in addition, the amount of computation is reduced and the adaptability to the environment is enhanced. Because BP neural network is optimized based on gradient descent, it has the disadvantages of slow convergence and is easy to fall into local minima and sensitive to the initial weight and bias of the network. In this paper, the PSO (particle swarm optimization) algorithm is improved to optimize the BP neural network, which effectively improves the convergence speed of the algorithm and enhances the capabilities of the global and local search. The upper triangle part of the covariance matrix of the received data is selected as the input of the network, and IPSO-BP neural network model is applied to estimate the position of the targets.

The rest of the paper is organized as follows. In Section 2, the basic FDA model is described and the basic criterion is derived for the FDA-BP through mathematical analysis. In Section 3, the FDA-BP model is optimized by using IPSO. In Section 4, the simulation analysis in MATLAB data and FEKO data is performed.

\section{Signal Model}

2.1. Model of FDA. The FDA model is shown in Figure 1. Unlike the conventional phased arrays, FDA has a small

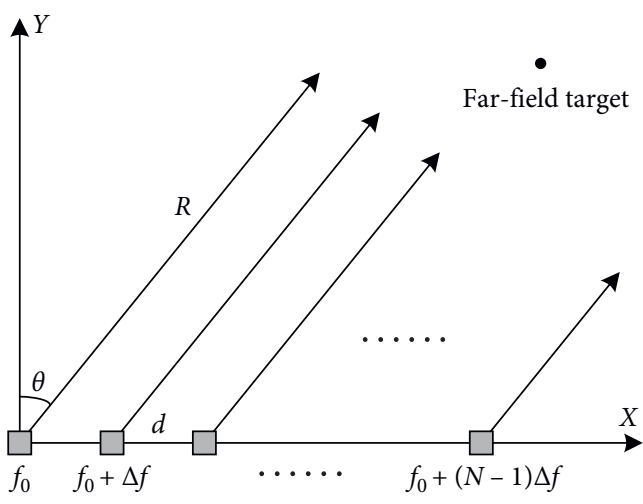

Figure 1: The model of frequency diverse array.

frequency increment between two adjacent array elements [18]. The transmission frequency $f_{n}$ of the $n$-th array element is shown as follows:

$$
f_{n}=f_{0}+(n-1) \Delta f, \quad n=0,1, \ldots, N-1,
$$

where $\Delta f$ is the frequency increment with reference to the carrier frequency $f_{0}$ and $N$ is the number of array elements. The transmitting signal of the $n$-th element is represented as $s_{n}(t)=a(t) e^{j 2 \pi f_{n} t}$ with the complex envelope $a(t)$. The single-frequency receiving FDA mode is adopted with the first array element as a reference, where each array element forms a transmitting and receiving channel and only receives the echo signal from itself; the echo signal of the $n$-th array element is expressed as [19]

$$
\begin{aligned}
y_{n}(t)= & a\left(t-\frac{2 R_{k}}{c}+\frac{2 \mathrm{nd} \sin \theta_{k}}{c}\right) \\
& \exp \left\{j 2 \pi f_{n}\left(t-\frac{2 R_{k}}{c}+\frac{2 \mathrm{nd} \sin \theta_{k}}{c}\right)\right\}+n_{n}(t),
\end{aligned}
$$

where $n_{n}(t)$ denotes the additive noise and is uncorrelated with signal, $\theta_{k}$ and $R_{k}$ are the azimuth angle and the distance of the $k$-th target, respectively, $d$ is the spacing of the array elements, and $c$ is the speed of light. Due to the narrow bandwidth signal and slow amplitude change, the difference of the envelope of each element can be ignored [20]. Therefore, the equation $a\left(t-\left(2 R_{k} / c\right)+\left(2 \mathrm{nd} \sin \theta_{k} / c\right)\right)=x_{k}(t)$ is obtained. Through matching filtering and down frequency conversion processing [21], the signal model can be expressed as

$$
\mathbf{Y}(t)=\mathbf{A}(R, \theta) \mathbf{X}(t)+\mathbf{N}(t)
$$

where the received signal vector is $\mathbf{Y}(t)=\left[y_{0}(t), y_{1}(t), \ldots\right.$, $\left.y_{N-1}(t)\right]^{\mathrm{T}}$, the source vector of targets is $\mathbf{X}(t)=\left[x_{1}(t)\right.$, $\left.x_{2}(t), \ldots, x_{K}(t)\right]^{\mathrm{T}}$, the noise vector is $\mathbf{N}(t)=\left[n_{0}(t)\right.$, $\left.n_{1}(t), \ldots, n_{N-1}(t)\right]^{\mathrm{T}}$, and $K$ is the number of targets. The steering vector is described as

$$
\mathbf{A}(R, \theta)=\left[\mathbf{a}\left(R_{1}, \theta_{1}\right), \mathbf{a}\left(R_{2}, \theta_{2}\right), \ldots, \mathbf{a}\left(R_{K}, \theta_{k}\right)\right],
$$

where $\mathbf{a}\left(R_{k}, \theta_{k}\right)=\left[a_{0}\left(R_{k}, \theta_{k}\right), a_{1}\left(R_{k}, \theta_{k}\right), \ldots, a_{N-1}\left(R_{k}, \theta_{k}\right)\right]^{\mathrm{T}}$, $a_{n}\left(R_{k}, \theta_{k}\right)=\exp \quad\left\{j 2 \pi\left(f_{0}+n \Delta f\right) \quad\left(-\left(2 R_{k} / c\right)+(2\right.\right.$ nd $\sin$ 
$\left.\left.\left.\theta_{k} / c\right)\right)\right\}$. The main task of this paper is to determine the angle and distance according to equation (3) by using the BP network.

The space-frequency covariance matrix $\mathbf{R}$ is estimated with the definition as follows:

$$
\mathbf{R}=E\left[\mathbf{Y}(t) \mathbf{Y}^{H}(t)\right]
$$

The upper triangular matrix of $\mathbf{R}$ consisting of $N(N-$ 1)/2 elements is chosen to construct the input vector of the model as follows [22]:

$$
\mathbf{R}_{1}=\left[R_{12}, R_{13}, \ldots, R_{1 N}, R_{23}, R_{24}, \ldots, R_{(N-1) N}\right]^{\mathrm{T}} .
$$

Meanwhile, the real and imaginary parts of each element in the vector $\mathbf{R}_{1}$ are separated to form a $N(N-1) \times 1$-dimensional vector $\mathbf{R}_{2}$, and the input set of the BP network is expressed as

$$
\mathbf{R}_{\text {in }}=\left[\mathbf{R}_{2}^{1}, \mathbf{R}_{2}^{2}, \mathbf{R}_{2}^{3}, \ldots, \mathbf{R}_{2}^{k}, \ldots, \mathbf{R}_{2}^{K}\right],
$$

where $\mathbf{R}_{\text {in }}$ is a $N(N-1) \times K$-dimensional matrix.

2.2. Model of FDA-BP. A typical BP neural network model of FDA radar (FDA-BP) is shown in Figure 2 with threelayer neuron operating [23], the input of the FDA-BP model is $\mathbf{R}_{\mathrm{in}}$, and the output is the estimates of the azimuth angle and the distance of targets. Every neuron in each layer is connected to every neuron in the adjacent forward layer, and no connections are permitted between the neurons belonging to the same layer. Each neuron is characterized by a transfer function and bias, and each connection between two neurons by a weight vector. Weight coefficients are typically adjustable and calculated by means of an adaptive algorithm combined with training samples (input-output signals) presented during the training (or learning) phase.

Signals propagate gradually through the network from the input layer and the hidden layers up to the output layer. The outputs of the hidden layer and output layer [23], $H_{h}$ and $o_{l}=\left(\widehat{\theta}_{l}, \widehat{R}_{l}\right)$, can be, respectively, written as

$$
\begin{gathered}
H_{h}=f\left(\sum_{i=1}^{M} w_{i h}-a_{i h}\right), \quad h=1,2, \ldots, Q, \\
o_{l}=\sum_{h=1}^{Q} H_{h} w_{h l}-b_{h l}, \quad l=1,2, \ldots, L,
\end{gathered}
$$

where $M, Q$, and $L$ are the numbers of neurons in the input layer, the hidden layer, and the output layer, respectively, $w_{i h}$ and $a_{i h}$ are the weight coefficient and bias value between input layer and hidden layer, respectively, and $w_{h l}$ and $b_{h l}$ are the weight matrix and bias value between hidden layer and output layer. Function $f(\cdot)$ is the activation function of each neuron and linear for input and output layers but is sigmoid (tan-sigmoid in the particular case) for hidden layer as follows:

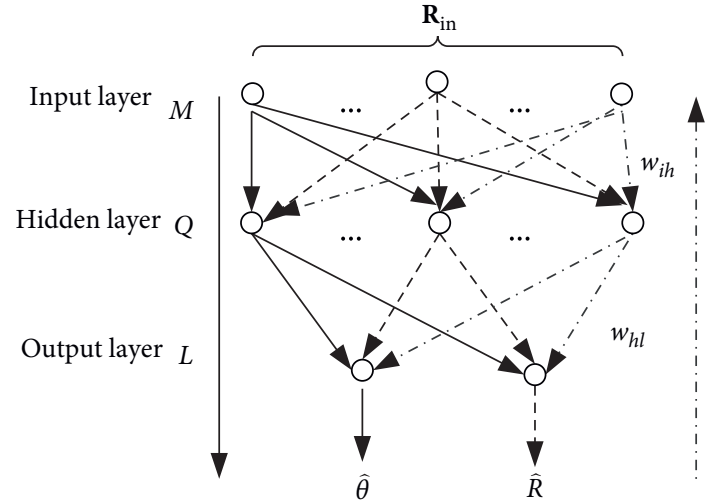

Figure 2: The model of BP neural network.

$$
f(u)=\frac{1}{1+e^{-u}}
$$

In order to obtain the weight coefficients and bias values, the training process is essential. The input vectors are presented to the input neurons and output vectors are calculated. The errors of estimates are computed as follows:

$$
e_{l}=\left(\theta_{l}, R_{l}\right)-\left(\widehat{\theta}_{l}, \widehat{R}_{l}\right), \quad l=1,2, \ldots, L .
$$

The error $e_{l}$ is backward-propagated, and the weights and bias values from the output layer to the hidden layer are updated by

$$
\begin{aligned}
w_{h l} & =w_{h l}+\eta H_{h} e_{l}, \\
b_{h l} & =b_{h l}+e_{l},
\end{aligned}
$$

where $\eta$ is the learning rate of the network. Similarly, the weights and bias values from the hidden layer to the input layer are updated by

$$
\begin{aligned}
& w_{i h}=w_{i h}+\eta H_{h}\left(1-H_{h}\right) R_{\mathrm{in}}(i) \sum_{l=1}^{L} w_{h l} e_{l}, \\
& a_{i h}=a_{i h} \eta H_{h}\left(1-H_{h}\right) R_{\mathrm{in}}(i) \sum_{l=1}^{L} w_{h l} e_{l} .
\end{aligned}
$$

The training process proceeds until the errors are lower than the prescribed values, or the maximum number of iterations is reached.

As the BP neural network algorithm is based on gradient, it has the disadvantages of slow convergence and is easily trapped into the local optimal solution and sensitive to the value of weight and biases during network initialization [24]. PSO algorithm is introduced to FDA-BP model, which has better robustness and global search ability based on the swarm intelligence theory. The improved PSO algorithm is applied to optimize the weights and biases of FDA-BP network to form an FDA-BP-IPSO algorithm in the next section.

\section{FDA-IPSO-BP Algorithm}

3.1. Problem of the Standard PSO Algorithm. The inertia weight PSO algorithm was proposed in [24], the essence of which is random search. Every particle in the swarm is 
considered to be massless and un-volumetric, and the process of optimization is carried out with the basis of particles' position and speed. Assuming that there exists a particle swarm in Q-dimensional space, which consists of $m$ particles, the position and speed of the $i$-th particle are given by

$$
\begin{aligned}
& X^{i}=\left(x_{i, 1}, x_{i, 2}, \ldots, x_{i, Q}\right), \\
& V^{i}=\left(v_{i, 1}, v_{i, 2}, \ldots, v_{i, Q}\right) .
\end{aligned}
$$

The particle swarm will get its own optimal solution and acquire the global optimal solution during each iteration, which is denoted as $P^{i}=\left(p_{i, 1}, p_{i, 2}, \ldots, p_{i, Q}\right)$. The position and speed are updated, respectively, according to the following equations:

$$
\begin{aligned}
v_{i, j}(t+1)= & w \times v_{i, j}(t)+c_{1} \times r_{1} \\
& \times\left[p_{i, j}-x_{i, j}(t)\right]+c_{2} \times r_{2} \times\left[p_{g, j}-x_{i, j}(t)\right], \\
x_{i, j}(t+1)=x_{i, j}(t)+v_{i, j}(t+1), & j=1,2, \ldots, Q, \\
& i=1,2, \ldots, m,
\end{aligned}
$$

where $w$ denotes the inertia weight, $c_{1}$ and $c_{2}$ are learning factors, $r_{1}$ and $r_{2}$ are random numbers in the range of $[0,1]$, and $p_{g, j}$ is the global optimal value. If $v_{i, j}>V_{\max }$, we get $v_{i, j}=V_{\max }$, while $v_{i, j}<-V_{\max }, v_{i, j}=-V_{\max }$, where $V_{\max }$ is the maximum speed limit value.

For the above analysis, the inertia weight is an important parameter to control the deviation of particles movement from global solution. In general, it is linearly updated with the expression $w=w_{\max }-t \times\left(w_{\max }-w_{\min }\right) / t_{\max }$; therefore, the convergence speed of the inertia weight PSO algorithm is slow [25]. On the other hand, with the decrease of inertia weight value in the later stage, the global search ability becomes weak. Once the optimal position of particle swarm is not the optimal value of the objective function, the algorithm will fall into the precocity state, resulting in the lack of diversity and local optimization of the particle swarm. Therefore, in this paper, the inertia weight is nonlinearly modified to ensure the global optimization of the particle swarm, meanwhile improving the local search ability of the PSO algorithm, and avoiding the premature convergence.

3.2. Improved PSO Algorithm for the FDA-BP Model. A nonlinear weight $w^{\prime}$ is applied in equation (14), and the improved PSO (IPSO) algorithm in this paper is formed, which can improve the slow convergence and local optimization. The nonlinear weight $w^{\prime}$ is written as

$$
w^{\prime}=w_{\max }-\left(w_{\max }-w_{\min }\right) \times \tan \left(\frac{t}{t_{\max }} \times \frac{\pi}{4}\right),
$$

where $w_{\max }=0.9$ and $w_{\min }=0.4$ and $t$ and $t_{\max }$ are the numbers of the current iteration and maximum iteration. From equation (16), $w^{\prime}$ is close to $w_{\max }$ at the beginning of the iteration processing, which leads to the large step for global optimization. With the increase of the iteration number $t$, the weight $w^{\prime}$ decreases, which ensures the smaller step for local optimization.

In order to evaluate the performance of the proposed algorithm, Ackley fitness function is used to compare the convergence of standard PSO, inertia weight PSO, and IPSO algorithms. The expression of the Ackley fitness function is written as [26]

$$
\begin{aligned}
\min f(x)= & -20 \exp \left(-0.2 \sqrt{\frac{1}{n} \sum_{j=1}^{n} x_{j}^{2}}\right) \\
& -\exp \left(\frac{1}{n} \sum_{j=1}^{n} \cos \left(2 \pi x_{j}\right)\right) \\
& +22.71282, \quad x_{j} \in[-5,5], j=1,2, \ldots, n,
\end{aligned}
$$

where $x$ is the value of the function argument.

In this experiment, the weight of the PSO algorithm without the inertia weight (named as the standard PSO, abbreviated as PSO) is 1 , and that of the inertia weights PSO and IPSO is $w$ and $w^{\prime}$, respectively. The Ackley function has many local optimal traps and is an unconstrained optimization problem. When $n=2$, the global optimal solution is 0 . For the parameters of the three algorithms, the particle number is $m=20$, the learning factors are $c_{1}=c_{2}=1.49445$, $V_{\max }=1$, and $t_{\max }=100$. The fitness curves of the test function are shown in Figure 3; the $X$-axis is the number of evolution and the $Y$-axis is the fitness value. Compared with the first two algorithms, the IPSO algorithm has better global and local search ability to obtain the global optimal solution faster and more accurately. The IPSO method is combined with FAD-BP into FAD-IPSO-BP algorithm in this paper.

FAD-IPSO-BP algorithm improves the connection weights and thresholds in the standard BP neural network, which can reach the vicinity of the global optimal solution. In this vicinity region, the BP neural network with strong local search ability is used to perform the optimal value search. Therefore, the complete network model is established.

The implementation process of FAD-IPSO-BP algorithm can be summarized as follows. Firstly, the received data are generated by FDA radar, weights and offsets of the network are initialized, and a three-layer BP neural network is preliminarily constructed. Secondly, IPSO algorithm is introduced to optimize and train the weight coefficients of FDA-BP neural network. Finally, the azimuth and distance of the targets are estimated by the trained BP neural network.

In order to test the performance of the proposed algorithm, the simulation experiments in MATLAB and FEKO simulation software are performed in the next section.

\section{Simulation}

4.1. Estimation of Target Position Using MATLAB Simulation Data. In this section, two experiments shown in Table 1 are performed in the MATLAB simulation software. In 


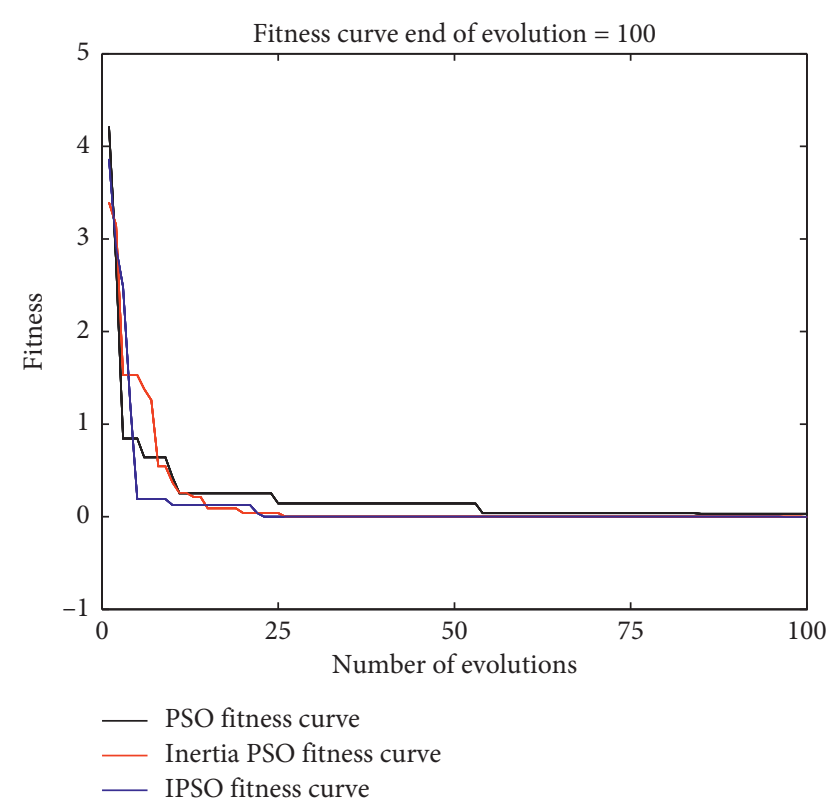

FIGURE 3: The fitness curves of PSO, inertia weights PSO, and IPSO algorithms.

experiment 1 , different targets are localized by using the FDA-IPSO-BP, FDA-BP, and FDA-MUSIC algorithms. In experiment 2 , multiple estimates are made using the FDAIPSO-BP and FDA-BP algorithms for targets $\left(5^{\circ}, 8 \mathrm{~km}\right)$, $\left(15^{\circ}, 8 \mathrm{~km}\right)$, and $\left(20^{\circ}, 12 \mathrm{~km}\right)$, respectively, under the different SNRs and snapshots.

In experiment 1, an FDA uniform linear array consists of 11 array elements, and the signal sources are selected as narrow-band signals with a reference carrier frequency of $f_{0}=10 \mathrm{GHz}$. The spacing of the array elements is $d=\lambda / 2$, the noise is independent zero-mean Gaussian white noise, the SNR is $10 \mathrm{~dB}$, and the number of snapshots is 100.24 different targets are utilized for positioning, where 8 angle parameters include $5^{\circ}, 10^{\circ}, \ldots, 40^{\circ}$ with the interval $5^{\circ}$, and 3 distance parameters are $4 \mathrm{~km}, 8 \mathrm{~km}, 12 \mathrm{~km}$, respectively. The different angles and distances are constituted into 24 target positions.

The 240 sets of data as input data $\mathbf{R}_{\text {in }}$ for the network are obtained from each of the above 24 targets calculated 10 times in equation (7), of which 216 sets obtained from the first 9 calculations of the 24 targets are selected as input data for network training period and 24 sets of data from the last calculation are selected as predictive input data to estimate the azimuth and range of the targets as stated above. According to the dimension of vector $\mathbf{R}_{2}$, the number of neurons in the input layer is $N(N-1)=11 \times 10=110$. In order to reduce the complexity of neural network, PCA (principal component analysis) algorithm is used for dimensionality reduction of $\mathbf{R}_{2}$, and the number of neurons in the input layer is selected as 14 through comprehensive consideration of the calculation ability of MATLAB software and complexity of neural network. The output is two parameters (distance and angle), and the output layer is composed of two neurons. The number of neurons in the hidden layer is calculated according to the empirical formula, $Q=\sqrt{M+L}+\alpha$, where $Q, M$, and $L$ are the numbers of the neurons in hidden layer, input layer, and output layer, respectively, and $\alpha$ is a constant of $[0,10]$. In this paper, $M=14, L=2$, and $\alpha=7$; then, $Q=11$. The same treatment is used in Section 4.2.

Under the condition of the single-frequency receiving FDA mode, the FDA-MUSIC algorithm requires two frequency increments, $\Delta f_{1}=5 \mathrm{kHz}$ and $\Delta f_{2}=-5 \mathrm{kHz}$, to decouple the azimuth and range of the targets, and the target position is obtained by using two-dimensional spectral peak search. However, the FDA-IPSO-BP and FDA-BP algorithms only need a single frequency increment, $\Delta f_{1}=5 \mathrm{kHz}$, to determine the localization of target, which is an obvious advantage of the two algorithms. The experimental results are shown in Figure 4, where $X$-axis and $Y$ axis represent the angle and the range, respectively.

The average errors are shown in Table 2, where the range of two-dimensional spectrum peak search angle in FDAMUSIC algorithm is from $0^{\circ}$ to $180^{\circ}$ with the interval $6^{\circ}$ and the distance from $3 \mathrm{~km}$ to $15 \mathrm{~km}$ with the interval $50 \mathrm{~m}$. The network training errors of FDA-IPSO-BP algorithm and FDA-BP algorithm are set to $1 \mathrm{e}-3$. From Figure 4 and Table 1, it can be seen that the FDA-IPSO-BP algorithm has high target localization accuracy.

In experiment 2 , the performance of the FDA-IPSO-BP algorithm and the FDA-BP algorithm will be numerically compared through the root mean square error (RMSE) with the expression as follows:

$$
\begin{gathered}
\operatorname{RMSE}_{\theta}=\sqrt{\frac{1}{H \times K} \sum_{k=1}^{K} \sum_{h=1}^{H}\left(\theta_{k}-\widehat{\theta}_{k h}\right)^{2}}, \\
\operatorname{RMSE}_{R}=\sqrt{\frac{1}{H \times K} \sum_{k=1}^{K} \sum_{h=1}^{H}\left(R_{k}-\widehat{R}_{k h}\right)^{2}},
\end{gathered}
$$

where $H$ is the number of Monte Carlos, $\widehat{\theta}_{k h}$ and $\widehat{R}_{k h}$ are the estimates of the $h$-th experiment of the angle and distance, respectively, and $\theta_{k}$ and $R_{k}$ are the actual values of the $k$-th source parameters of the angle and the distance.

The network structure and the training sets of FDAIPSO-BP algorithm and FDA-BP algorithm are consistent with experiment 1 . The target positions are selected as $\left(5^{\circ}, 8 \mathrm{~km}\right),\left(15^{\circ}, 8 \mathrm{~km}\right)$, and $\left(20^{\circ}, 12 \mathrm{~km}\right)$, and the accuracy of target position estimation is compared by changing the signal-to-noise ratio and the number of snapshots of the algorithm, respectively.

The number of snapshots is fixed at 100 , SNR value varies from $-5 \mathrm{~dB}$ to $15 \mathrm{~dB}$ with the step $5 \mathrm{~dB}$, and the Monte Carlo number is 700 . The total 2100 test sets are computed for the three targets with 700 times for each one in equation (7). The root mean square error (RMSE) of these test sets is calculated using equations (18) and (19) between the estimates and the true location of the targets. Finally, the averages of the RMSE of the three targets are calculated and shown in Figures 5 and 6, where the $X$-axis 
TABLE 1: The simulation experiments of target location.

\begin{tabular}{lcccc}
\hline & $\begin{array}{c}\text { The number of } \\
\text { targets }\end{array}$ & Algorithm & Performance index & Experimental scene \\
\hline Experiment 1 & 24 & FDA-IPSO-BP, FDA-BP, FDA- & Average error & $\begin{array}{c}f_{0}=10 \mathrm{GHz}, \mathrm{SNR}=10 \mathrm{~dB}, \\
\text { The number of snapshots }=100 \\
f_{0}=10 \mathrm{GHz}, \mathrm{SNR}=(-5: 5: 15) \mathrm{dB}, \\
\text { Experiment } 2\end{array}$ \\
& 3 & MUSIC & The root mean square \\
error & $\begin{array}{c}\text { Ther of snapshots }=100: 100: \\
600\end{array}$ \\
\hline
\end{tabular}

TABLE 2: The average error of the three algorithms.

\begin{tabular}{lccc}
\hline & FDA-IPSO-BP & FDA-BP & FDA-MUSIC \\
\hline Average error of 24 target angles $\left(^{\circ}\right)$ & 0.269 & 0.334 & 4.833 \\
Average error of 24 target distances $(\mathrm{m})$ & 75.000 & 185.250 & $1.837 e 3$ \\
\hline
\end{tabular}

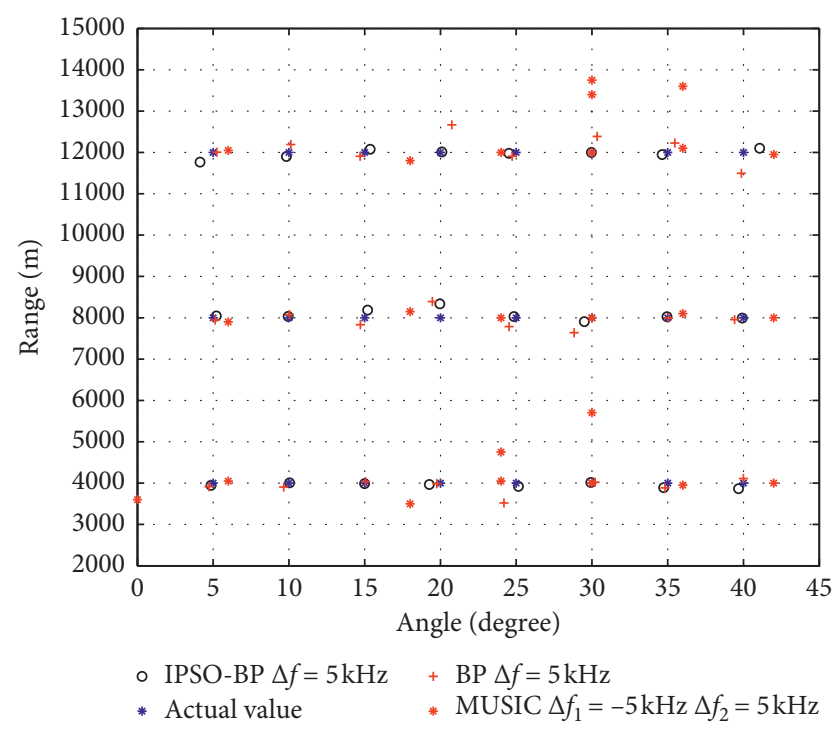

Figure 4: Target localization result of the three algorithms.

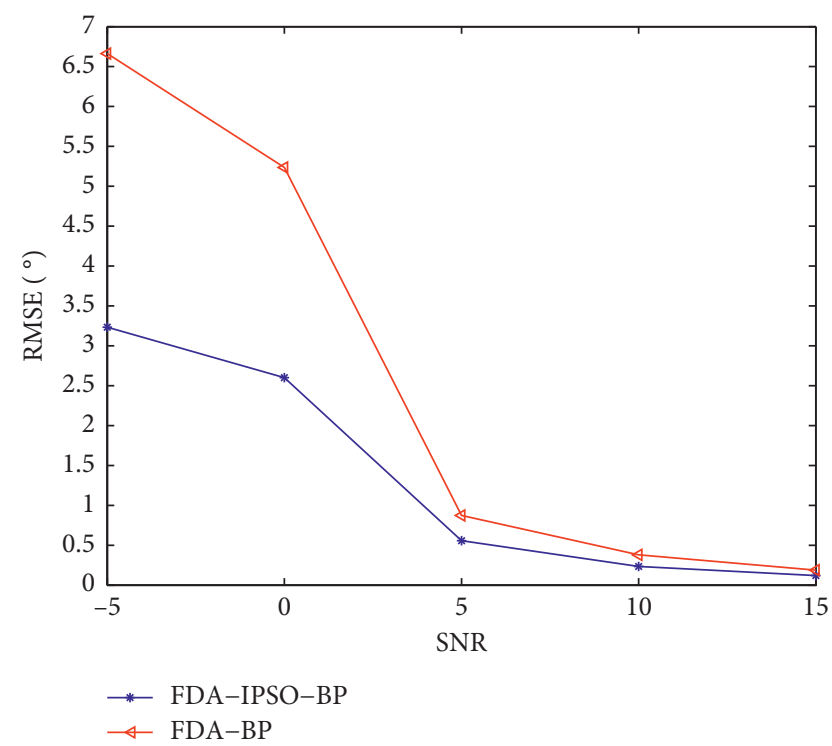

FIGURE 5: RMSE of azimuth estimation versus SNR.

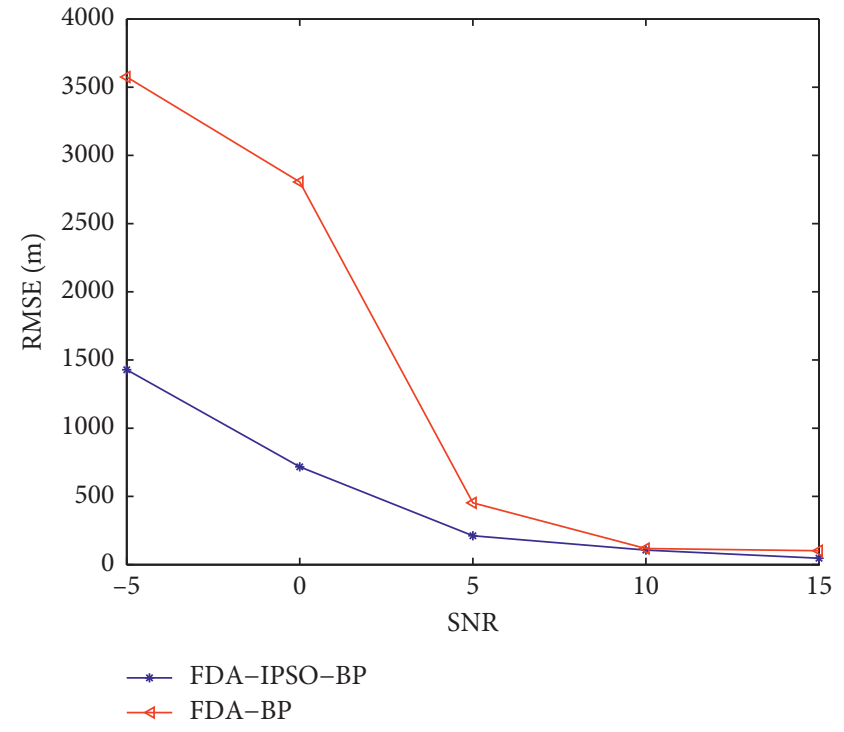

FIGURE 6: RMSE of range estimation versus SNR.

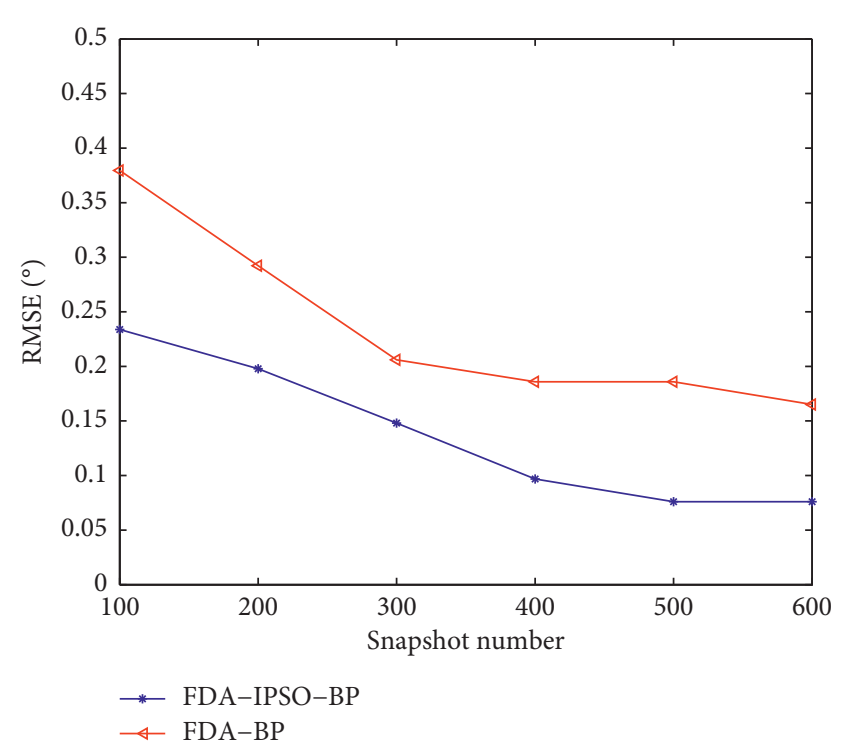

FIGURE 7: RMSE of azimuth estimation versus snapshot. 


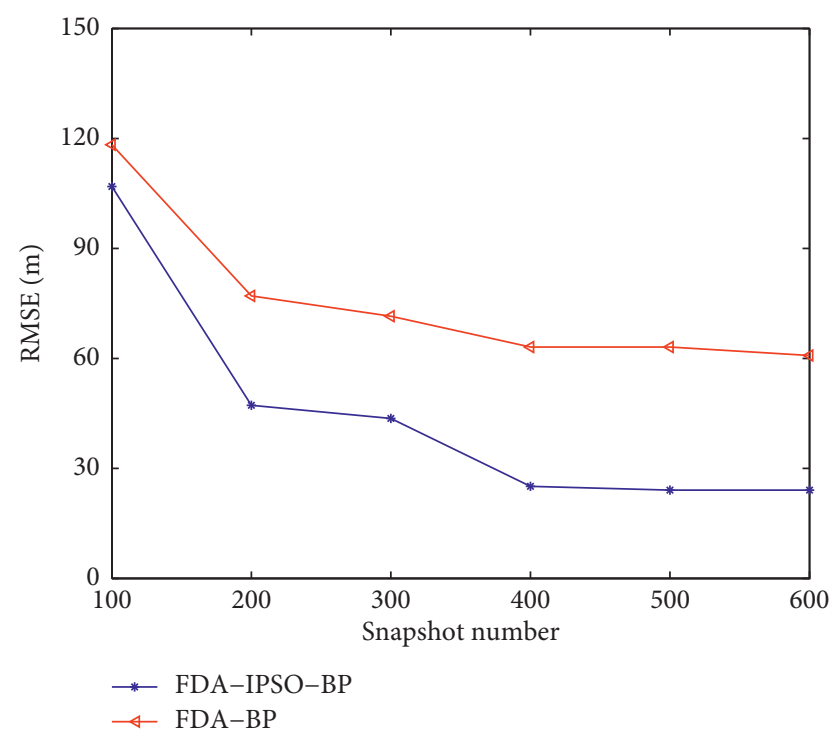

FIGURE 8: RMSE of range estimation versus snapshot.

indicates SNR and the $Y$-axis represents the RMSE of the azimuth and range, respectively.

Similarly, the average of the RMSE for the three targets is calculated by varying the number of snapshots shown in Figures 7 and 8 , where the SNR is fixed at $10 \mathrm{~dB}$, the number of snapshots is constantly changing from 100 to 600 with the step 100, and the Monte Carlo number is 700 .

From the above experiment results from Figures 5-8, it can be seen that the FDA-IPSO-BP algorithm has higher estimation accuracy in azimuth and distance than the FDA$\mathrm{BP}$ algorithm, where the IPSO method is utilized to prevent BP network from falling into local optimum as much as possible.

\subsection{Estimation of Target Position Using Data from FEKO} Simulation Software. In experiment 3, FEKO simulation data is used to further verify the application of FDA-IPSO$\mathrm{BP}$ close to the actual environment. FEKO software is a powerful three-dimensional full-wave electromagnetic simulation software. As shown in Figure 9, a 7-array FDA radar is constructed in FEKO. The target is a rectangular sheet of 1 square meter. The initial carrier frequency of the array is $f_{0}=1 \mathrm{GHz}$, and the frequency deviation is $\Delta f=1050 \mathrm{kHz}$.

The received data obtained by moving the location of the target are used as samples for this experiment at angles from $25.0^{\circ}$ to $35.0^{\circ}$ with interval $0.1^{\circ}$, and distances from $95.0 \mathrm{~m}$ to $105.0 \mathrm{~m}$ with interval $0.1 \mathrm{~m}$. Considering the operation time of the FEKO software to generate simulation data, the training data are obtained from the 101 target positions composed of the one-to-one point matching between the angles and distances. The 101 target position set is $\left\{\left(25.0^{\circ}, 95.0 \mathrm{~m}\right), \quad\left(25.1^{\circ}, 95.1 \mathrm{~m}\right),\left(25.2^{\circ}, 95.2 \mathrm{~m}\right), \ldots,\left(35.0^{\circ}\right.\right.$, $105.0 \mathrm{~m})\}$. The received data from the target position $\left(30^{\circ}, 100 \mathrm{~m}\right)$ are used as an input sample for the testing period.
TABLE 3: Sample results of FEKO simulation.

\begin{tabular}{lc}
\hline Input data & 101 samples \\
\hline Actual target location & $\left(30^{\circ}, 100 \mathrm{~m}\right)$ \\
Target location estimates & $\left(29.997^{\circ}, 99.998 \mathrm{~m}\right)$ \\
\hline
\end{tabular}

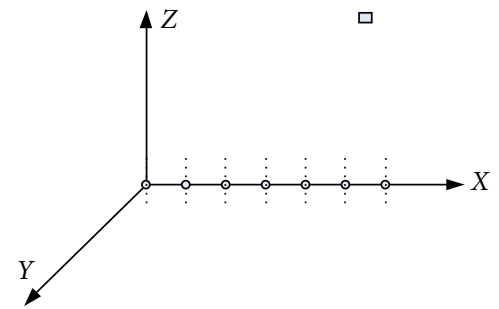

FIGURE 9: The model of antenna array in the FEKO simulation environment.

The numbers of neurons in the input layer, the hidden layer, and the output layer are 14,11, and 2, respectively. Because there is no signal-to-noise ratio parameter setting option in FEKO software, we add the white noise to the received data from $\mathrm{FEKO}$ software with $\mathrm{SNR}=15 \mathrm{~dB}$.

The azimuth and distance information estimates are shown in Table 3. As can be seen from Table 3, the target position obtained by the FDA-IPSO-BP algorithm matches the actual target. The proposed algorithm avoids the coupling problem of angle and distance and saves time and computing resources to a large extent compared with MUSIC-type algorithms.

\section{Conclusion}

In order to improve the precision and robustness of target localization in frequency diverse array (FDA) radar, the BP neural network is merged with FDA radar, and the improved particle swarm optimization (IPSO) algorithm nonlinearly updates the weights to keep away from local optimum and sensitivity to initialization of weights in this paper. Furthermore, the angle and distance are decoupled by single frequency increment. It can be seen from the experiment results under MATLAB and FEKO simulation environments that the proposed method has an effective improvement in the accuracy of target localization, compared with FDA-BP and FDA-MUSIC algorithms. The future work is to build experimental platform and use the proposed algorithm to achieve radar target positioning with the actual measurement data in the near-field and far-field environment.

\section{Notations}

$(\cdot)^{\mathrm{T}}$ : Matrix transpose

$(\cdot)^{\mathrm{H}}$ : Matrix conjugate transpose.

\section{Data Availability}

The data used to support the findings of this study are available from the corresponding author upon request. 


\section{Conflicts of Interest}

The authors declare that they have no conflicts of interest.

\section{Acknowledgments}

This work was supported by the National Natural Science Foundation of China (grant nos. 61861011 and 51808554), the Guangxi Natural Science Foundation (grant nos. 2017GXNSFAA198050 and 2018GXNSFAA138091), the Science and Technology on Near-Surface Detection Laboratory Foundation (grant no. TCGZ2017A010), and Major Science and Technology Foundation of Guangxi Province (grant no. AA17204093).

\section{References}

[1] P. Antonik, W. C. Wicks, H. D. Griffiths et al., "Frequency diverse array radars," in Proceedings of the IEEE Radar Conference, pp. 470-475, Verona, NY, USA, April 2006.

[2] J. Huang, K. F. Tong, and C. J. Bake, "Frequency diverse array with beam scanning feature," in Proceedings of the 2008 IEEE Antennas and Propagation Society International Symposium, pp. 1-4, IEEE, San Diego, CA, USA, July 2008.

[3] J. Huang, K. F. Tong, K. Woodbridge et al., "Frequency diverse array: simulation and design," in Proceedings of the Antennas and Propagation Conference, pp. 1-4, IEEE, Pasadena, CA, USA, May 2009.

[4] P. Baizert, T. B. Hale, M. A. Temple, and M. C. Wicks, "Forward-looking radar GMTI benefits using a linear frequency diverse array," Electronics Letters, vol. 42, no. 22, pp. 1311-1312, 2006.

[5] J. Farooq, M. A. Temple, and M. A. Saville, "Application of frequency diverse arrays to synthetic aperture radar imaging," in Proceedings of the International Conference on Electromagnetics in Advanced Applications, pp. 447-449, IEEE, Torino, Italy, September 2007.

[6] A. Basit, I. M. Qureshi, W. Khan et al., "Beam pattern synthesis for a cognitive frequency diverse array radar to localize multiple targets with same direction but different ranges," in Proceedings of the 13th International Bhurban Conference on Applied Sciences and Technology (IBCAST), pp. 682-688, Islamabad, Pakistan, January 2016.

[7] P. F. Sammartino, C. J. Baker, and H. D. Griffiths, "Rangeangle dependent waveform," in Proceedings of the IEEE National Radar Conference-Proceedings, pp. 511-515, IEEE, Washington, DC, USA, May 2010.

[8] L. Zhuang and X. Liu, "Application of frequency diverse to suppress grating lobes in coherent MIMO radar with separated subapertures," EURASIP Journal on Advances in Signal Processing, vol. 2009, no. 1, pp. 1-10, 2009.

[9] K. V. Shanbhag, D. Deb, and M. Kulkarni, "MIMO radar with spatial-frequency diverse for improved detection performance," in Proceedings of the IEEE International Conference on Communication Control and Computing Technologies, pp. 66-70, IEEE, Ramanathapuram, India, October 2010.

[10] P. C. Gong, G. Liu, H. Huang et al., "Multidimensional parameter estimation method based on sparse iteration in FDAMIMO rada," Journal of Radars, vol. 7, no. 2, pp. 194-201, 2018.
[11] H. Chen and H. Shao, "Sparse reconstruction based target localization with frequency diverse array MIMO radar," in Proceedings of the 2015 IEEE China Summit and International Conference on Signal and Information Processing (ChinaSIP), pp. 94-98, Chengdu, China, July 2015.

[12] P. F. Sammartino, C. J. Baker, and H. D. Griffiths, "Frequency diverse MIMO techniques for radar," IEEE Transactions on Aerospace and Electronic Systems, vol. 49, no. 1, pp. 201-222, 2013.

[13] C. Cui, J. Xu, R. Gui et al., "Search-free DOD, DOA and range estimation for bistatic FDA-MIMO radar," IEEE Access, vol. 6, pp. 15431-15445, 2018.

[14] Z. Ping, "DOA estimation method based on neural network," in Proceedings of the 2015 10th International Conference on P2P, Parallel, Grid, Cloud and Internet Computing (3PGCIC), pp. 828-831, Krakow, Poland, November 2015.

[15] A. Faye, A. B. Youm, and J. D. Ndaw, "LVQ based DOA estimation," in Proceedings of the 2013 Fifth International Conference on Computational Intelligence, Communication Systems and Networks, pp. 245-250, IEEE, Madrid, Spain, June 2013.

[16] A. Faye, J. D. Ndaw, and A. S. Maiga, "Two-dimensional DOA estimation based on a single uniform linear array," in Proceedings of the 2017 25th Telecommunication Forum (TELFOR), pp. 1-4, Belgrade, Serbia, November 2017.

[17] J. D. Ndaw, A. Faye, and A. S. Maiga, "Decoupled 2D DOA estimation using LVQ neural networks and UCA arrays," in Proceedings of the 2016 IEEE Radio and Antenna Days of the Indian Ocean (RADIO), pp. 1-2, St. Gilles-les-Bains, Reunion, October 2016.

[18] Q. Liu, C. Jiang, L. Jin, and S. Ouyang, "Detection of subsurface target based on FDA-MIMO radar," International Journal of Antennas and Propagation, vol. 2018, Article ID 8629806, 8 pages, 2018.

[19] L. Huang, Z. M. He, X. Li et al., "Frequency diverse array radar for range-angle estimation based on difference co-array," Journal of System Simulation, vol. 29, no. 4, pp. 886-893, 2017.

[20] Z. Xiang and B. X. Chen, "Range-angle decoupled transmit beamforming with frequency diverse array," Journal of $R a$ dars, vol. 7, no. 2, pp. 212-219, 2018.

[21] J. W. Xu, L. Lan, G. S. Liao et al., "Range-angle matched receiver for coherent FDA radars," in Proceedings of 2017 IEEE Radar Conference, pp. 324-328, Seattle, WA, USA, May 2017.

[22] F. Y. Sun, Y. B. Tian, and Z. L. Ren, "DOA estimation with support vector machine based on hybrid kernel," Telecommunication Engineering, vol. 56, no. 3, pp. 302-307, 2016.

[23] M. Agatonovic, Z. Stankovic, I. Milovanovic et al., "Efficient neural network approach for 2D DOA estimation based on antenna array measurements," Progress In Electromagnetics Research, vol. 137, pp. 741-758, 2013.

[24] N. Kumar and S. Kumar Sharma, "Inertia weight controlled PSO for task scheduling in cloud computing," in Proceedings of the 2018 International Conference on Computing, Power and Communication Technologies (GUCON), pp. 155-160, Greater Noida, India, September 2018.

[25] H. L. Tao, X. P. Li, S. Z. Zhang et al., "Forecast of railway passenger traffic volume based on IPSO-BP neural network," Railway Transport And Economy, vol. 9, pp. 82-86, 2011.

[26] C. J. Wen, B. Xia, and X. Liu, "Solution of second order ackley function based on SAPSO algorithm," in Proceedings of the 2017 IEEE 3rd International Conference on Control Science and Systems Engineering, pp. 624-627, Beijing, China, August 2017. 